Euskal ikerketen aldizkaria | Revue d'études basques |

Revista de estudios vascos | Basque studies review

$6 \mid 2001$

Numéro VI

\title{
La précarité des journalistes locaux
}

\section{Eguzki Urteaga}

\section{OpenEdition \\ Journals}

Édition électronique

URL : http://journals.openedition.org/lapurdum/1243

DOI : 10.4000/lapurdum.1243

ISSN : 1965-0655

Éditeur

IKER

Édition imprimée

Date de publication : 1 octobre 2001

Pagination : 369-389

ISBN : 2-84127-156-0

ISSN : $1273-3830$

Référence électronique

Eguzki Urteaga, «La précarité des journalistes locaux», Lapurdum [En ligne], 6 | 2001, mis en ligne le 01 juin 2009, consulté le 31 janvier 2020. URL : http://journals.openedition.org/lapurdum/1243 ; DOI 10.4000/lapurdum. 1243 


\section{Eguzki Urteaga 1}

\section{La précarité des journalistes locaux}

\section{Introduction}

La littérature sociologique s'est peu intéressée aux journałistes locaux. La faible documentation existante sur ce sujet est révèlatrice du manque de considération portée à ces acteurs ayant davantage suscité le mépris que la curiosité. La raison en incombe notamment au tait que ces chercheurs, don Wolton $^{2}$. lisent, rcgardent et écoutent peu ces professionnels et leurs titres d'appartenance. Toutefois, c'est de moins en moins le cas car ils ne peuvent les ignorer plus longtemps tant par leur nombre que par la diffusion de leurs médias largement supérieure à celle des organes de presse nationaux que d'aucuns qualifient de parisiens.

C'est pourquoi, depuis quelques années, des études concernent les journalistes de région privilégiant des questionnements divers. Certaines se sont penchées sur les rapports qu'ils entretiennent avec les notables en général et les élus locitux en particutier. I'attention est dès lors portic sur la fréquence, les lieux et les modalités des rencontres entre ces acteurs témoignant d'une connivence qui est mise en exergue atin de mieux saisir l'incidence qu'ont ces relations sur la manière dont les titres relatent les faits concernant le député-maire, le président du Conseil Général ou te conseiller régional. Influence qui est tantôt démontrée tantôt postulée. Ces travaux s'inscrivent dans une tradition visant à prouver la collusion entre les pouvoirs politique et médiatique.

D'autres travaux ont préféré attirer l'attention sur le rôle joué par les journalistes locaux et leurs médias dans le développement du sentiment d'insécurité voire de la violence elle-même. "Les représentations de l’insécurité sont façonnées par les médias. (...). De fait, la presse locale disqualifie constamment, et sans s'en rendre nécessairement compte, des quar-

\footnotetext{
${ }^{1}$ Eguzki Urteaga est Docteur en Sociologie de l'Université Victor Segalen Bordeaux Il et Licencié en Histoire mention Géographie de l'Université de Pau et des Pays de l'Adour. Après avoir effectué des recherches sur les mouvements sociaux et le militantisme, il a réalisé une Thèse de Doctorat intitulée Les journalistes locaux: mutation d'une profession sous la direction de François Dubet au sein du CADIS-CNRS. II a publié un ouvrage ayant pour titre Le motvement antimilitariste basque contemporain ainsi que plusieurs articles dans des revues universitaires dont La revista vasca de sociología y ciencia política : Inguruak et Giza eta gizarte zientzien aldizkaria : Uztaro. Actuellement, il travaille sur la théorie sociologique.
}

2 Wolton. D., Penser la communication. Paris, Flammarion, 1997. 
tiers ou des groupes qu'elle associe à la délinquance ou au crime, les donnant à voir sous l'angle principal du fait divers et de la menace". Cela "conforte des représentations qui amplifient la violence en flattant les préjugés de la population, mais parfois aussi en rendant compte avec objectivité des phénomènes observés". Et d'ajouter, "en conférant aux acteurs tentés par la violence une visibilité qu'ils n'atteignent pas autrement, les médias peuvent encourager, éventuellement malgré eux, des conduites spectaculaires" 3 .

En revanche, la condition de ces journalistes n'a guère piqué la curiosité des chercheurs car nous ne connaissons ni leurs carrières, ni leurs statuts. Tel est justement notre propos en examinant celle des journalistes professionnels dans les Pyrénées-Attantiques. À ce sujet, nous défendons l'hypothèse selon laquelle, depuis les années 70 , la condition journalistique locale se précarise ${ }^{4}$ peu à peu avec la faible progression des carrières, la fragilité de statuts et la modestie des revenus, bien que nous centrions notre analyse sur les deux premiers indicateurs. Plus précisément, à la promotion limitée des postes, des échelons et des fonctions s'ajoute la fragilité des statuts avec le développement des contrats précaires, du pigisme, du temps partiel comme du chômage pouvant précipiter momentanément ou durablement les journalistes dans l'exclusion sociale ${ }^{5}$. Cette précarité touche la profession dans sa totalité, malgré le fait que ses conséquences sont plus visibles et manifestes à ses lisières.

\section{Faible promotion des carrières}

\subsection{Progression de poste limitée}

En effet, au cours des années 80 et 90. l'évolution de postést réduite puisque la plupart des rédacteurs n'accèdent guère à l'encadrement, certains d'entre eux ne diviennent chef de service qu'après de nombreuses années d'attente et les rédacteurs en chef provicrnnent souvent de départements annexes. Rares sont ceux qui parviennent à la direction des médias locaux exigeant formation spécialisée, mobilité externe, quálification élevée et investissement supérieur. Même lorsque la possibilité leur en est offerte, ils la refusent, jugeant les responsabilités excessives, d'autant que le poids de la gestion humaine, administrative et financière ne leur laisse guère de temps pour le reportage.

L'accès à la direction illustre cette difficulté puisque peu nombreux sont les journalistes parvenant à ce poste aussi convoité que lointain, notamment dans les locales de Radio France, d'autant que multitude de directeurs sont issus de l'animation. Atteindre le poste de rédacteur en chef est également semé d'embûches dans la mesure où Sud Ouest, France 3. Radio France voire Pyrénées Presse préfèrent les accorder aux professionnels ayant œuvré en dehors de leur zone de diffusion. Ainsi, l'actuel rédacteur en chef de France 3 Pays Basque

\footnotetext{
3 Wieviorka. M., Violence en France. Paris, Seuil, 1999 p. 10.

4 Accardo. A., Journalistes précaires. Bordeaux, Le Mascaret, 1998.

5 Paugam. S., Le salarié de la précarité. Paris, PUF, 2000.
} 
provient de la locale de Tours, le rédacteur en chef de Radio Bleu Pau Béarn est issu de la région nantaise ou celui de Radio Bleu Pays Basque est originaire de Bordeaux. Le renouvellement des perceptions, des méthodes de travail et des hommes se fait au détriment du déroulement des carrières des autochtones. Dès lors, ces derniers se sentent souvent coincés, pris au piège, sans option véritable.

A l'exemple de la rédactrice de Radio Orthez 2001 qui y a débuté peu après sa création en juillet 1982 en faisant à la fois de l'animation et du journalisme. Son investissement lui a permis de bénéficier d'un contrat emploi solidarité (CES) en 1985 suivi d'autres contrats précaires. Cependant, il lui a fallu attendre 1989 pour obtenir un CDI en tant que rédactrice chargée de l'élaboration et de la présentation des journaux et, progressivement, ce rôle s'est transformé dans la mesure où elle assurait la formation comme la coordination des CES-CEC tout en occupant de la confection des plannings, des procranimes, de la comptabilité, des dossiers de candidature pour l'obtention de subventions. Pourtant. bien qu'exerçant le róli: de rédactrice en chef, elle s'est vue refuser ce rang par lá dircetion.

Les obstacles abondent tout autant pour devenir adjoint, la plupart des rédacteurs et des chefs de service n' ayant pas cette opportunité, tel ce professionnel : "J'ai débuté dans le journalisme en 1964 en tant que pigiste pour La Dépêche du Midi résidant dans les Hautes Pyrénées. En 1966, te titre m'a proposé le poste de rédacteur sportif à Lannemezan avant de m'intégrer en tant que journaliste polyvalent à la rédaction d'un quotidien départemental, s'appelant La Nouvelle République des Pyrénées, journal dont le siège se trouve à Tarbes. Très rapidement, j'ai été affecté au service des sponts, composé alors de deux membres, dont j'ai pris la direction à partir de 1973. A ce titre, j’avais la resfonsabilité des journalistes, des pigistes comme des correspondants sportifs. En 1993, suite au changement politique du Groupe La Dépêche du Midi auquel appartient le titre, j’ai accepté la proposition de Pyrénées Presse pour devenir chef du service des sports avec peu de chances de gravir les postes, d'autant que je suis en fin de carrière" 6 .

Les raisons de l'élévation restreinte de poste sont les suivantes. Premièrement, malgré la professionnalisation graduelle, un grand nombre de médias ne comprend guère d'encadrements chargés de gérer le budget, de nouer des partenariats, de représenter l'entreprise de presse ou d'animer l'équipe. La plupart des directions se trouvent au siège distant de nombreux kilomètres, à l'image des titres transfrontaliers. Certains organes de presse n'en disposent pas davantage pour d'autres motifs, à l'image d'Irulegiko Irratia. "Dès la création de la station en 1982, nous nous sommes opposés à la présence d'un directeur ayant une fonction gestionnaire et rédactionnelle par souci de préserver l'autonomie de la rédaction et d'assurer l'égalité entre journalistes en évitant tout rapport hiérarchique. La gestion est assurée par le bureau et par le Conseil d'Administration"?

${ }^{6}$ Entretien avec le chef du service des sports de Pyrénées Presse

7 Entretien avec un rédacteur de Radio Irouleguy: 
Deuxièmement, des journalistes ne parviennent souvent à ces postes qu'à la faveur de crises, à l'image de la directrice de Radio Oloron qui a fait ses débuts dans le journalisme à Oloron en 1987 suite à la décision de la station de recruter un professionnel. Elle était rédactrice chargée des reportages, du montage et de la présentation des bulletins d'information. Avec le temps, à l'aide de contrats précaires, la radio a fait appel à de nouveaux salariés n'ayant pas ou peu d'expérience du journalisme comme de la radiodiffusion. En 1992, elle accède à la direction de la station dans un contexte de crise qui risque de se solder par la vente de Radio Oloron à Chérie FM. Avec l'aide de deux personnes, elle la reprend en main en la dotant de nouveaux bureaux et Conseil d'Administration tout en leur asisinant des objectifs clairs. Sans cette crise, elle n'y serait sans doute jamais parvenue.

Troisièmement, l'absence de mobilité externe est en cause. En effet, les médias locaux en général et les quotidiens départementaux en particulier se caractérisent par une faible mobilité interne (entre agences) et surtout externe (entre médias) de leur personnel. Il n’est pas rare que des personnes fassent la totalité de leur carrière au sein des mêmes titre et service. Dès lors, ils n'ont pas été confrontés à d'autres sources, méthodes de travail, cultures d'entreprise qui leur permettent de s'ouvrir à de nouvelles pratiques professionnelles. Ce ne sont guère les personnes adéquates pour impulser une dynamique, insuffler de l'air frais et donner un nouveau souffle à des rédactions passablement engourdies. Or, c'est précisément ce que recherchuıl les PDG à défaut de faire signer une clause de mobilité. comme la pratique Sud Ouest depuis 1974

\section{2. Élévation d'échelon réduite}

L'avancement d'échelon connât des restrictions analogues puisque l'ancienneté dans l'entreprise de presse comme dans la profession ne suffit plus pour passer à l'échelon supérieur et devenir successivement journaliste spécialisé, chef d'édition voire grand reporter. Les médias s'efforcent d'y ralentir l'accès e/ou d'être plus sélectifs dans leur attribution. De ce fait, quelques chanceux connaissent une évolution relativement rapide alors que quantité d'autres sont condamnés à la base après vingt ans de carrière.

Ainsi, devenir grand reporter n'est pas tâche aisée compte tenu du fait que, dans l'ensemble du département, l'on n'en dénombre que quatre, chiffre inchangé au cours des dernières années. Cet échelon n'est plus exclusivement lié à l'ancienneté, mais la formation professionnelle ${ }^{8}$, l'investissement, la qualité du travail voire la dynannisation de l'équipe entrent également en jeu. Plus encore, la mobilité de l"encadrement entre médias et entre supports est telle que la discontinuité est de mise de sorte que l'effort opéré et les sacrifices consentis

8 Urteaga. E., "Kazetariak eta heziketa profesional iraunkurra". Bibo, Giza eta Gizarte Zientzien Aldizkaria: Uztaro, n`36, 2001, pp.53-71. 
ne reçoivent plus de récompense, le témoin de ce travail ayant quitté l'entreprise. En un mot, les journalistes ne récoltent plus les fruits de leur labeur.

De même, arriver à l'échelon "chef d'édition" n'a rien de systématique puisque le département n'en compte que six dont trois à France Bleu Béarn, un à France Bleu Pays Basque et un autre dar; chaque rédaction départementale de Sud Ouest. Pis encore, aucun rédacteur cuvrant dans les médias locaux et transfrontaliers n'y parvient. Devenir journaliste spécialisé est également ardu, parce que les organes de presse réfléchissent à deux fois avant d'accorder ce rang, sachant qu'une promotion précoce et généralisée donne lieu à une augmentation massive des dépenses. "Nous, les PDG, devons faire preuve de rigueur dans la gestion, en traquant les frais inutiles et les gaspillages sans revenir sur les acquis sociaux. L'un des metlleurs moyens consiste à retarder les élévations d'échelon du prorsonn.:" 9.

Cumment rendre compte de telles difficultés? En premier lieu, bien que l'ancienneté ne garancisse plus la progression d'échelon, gravir les marches suppose un certain âge. Les médias des Pyrénées-Atlantiques récompensent ainsi les années de fidèles et loyaux services, tel ces detıx grands reporters quinquagénaires. Par contre, nul bénéficiaire de cet échelon n'a moins de quarante ans. Le probleme réside dans le rajeunissement de la profession qui affecte l'ensemble des titres de telle sorte qu'il est ardu de devenir grand reporter. Tout au plus, les journalistes peuvent precipiter le processus.

En second lieu, l'affaiblissement syndical et le recul des adhésions auprès des "nouveaux journalistes" notamment ne sont pas sans effet, surtout dans le service public où les centrales syndicales jouent un rôle majeur lors des commissions paritaires regroupant les partenaires sociaux qui négocient les promotions du personnel. "Nous appuyons l'ensemble des candidatures, dont celles de la direction, ce qui ne nous empêche nullement de proposer nos candidat: at de nous battre jour qu'ils obticunent des avancements. Ainsi, grâce à mon action ct a leur travail, plusieurs journalistes de Radio Bleu Pays Basque ont connu des carrières plus rapides. Ils ont gravi les échelons en l'espace de quelques années, ce qui leur permet de jouir de salaires largement supérieurs au mien" 10.

En dernier lieu, cela est directement corrélé à la précarité des statuts car le pigisme fait disparaître les échelons de grand reporter, de chef d'édition et de journaliste spécialisé. N'étant pas titulaires, ils ne jouissent d'aucun avancement lié à l'ancienneté. Comme l'explique cet indépendant, "l'ancienneté est une donnée n'ayant aucun sens lorsque l'on est pigiste car le montant des piges est constant et ne progresse pas suivant le nombre d'années de collaboration. Résultat : l'on stagne par rapport à nous-mêmes et l'on régresse en relation aux journalistes intégrés dans des médias comme Sud Ouest, France Bleu Pays Basque ou France 3 Aquitaine. Les organes de presse locaux ou nationaux pour lesquels l'on travaille n'ont guère

9 Entretien avec le PDG de Pyrénées Presse.

10 Entretien avec un délégué syndical du SNI. 
I'intention de prendre en compte notre expérience el de la traduire dans un système d'échelons parallèle" 11.

\subsection{Avancée de fonction restreinte}

Une faible avancée, pour ne point parler de stagnation, est tout aussi perceptible entre fonctions dans la mesure où la transition de la photographie à la rédaction, du secrétariat de rédaction à la rédaction ou du laboratoire à la photographie est complexe, puisque les rédactions sont plus structurées et les services plus cloisonnés. Le rôle dévolu à chaque section se spécifie empêchant les transitions rapides et conséquentes.

Ainsi, être rédacteur relève du rêve pour nombre de secrétaires de rédaction, de journalistes reporter d'images (JRI) et de photographes. Rêve dont la réalisation s'éloigne. La dévalorisation des fonctions techniques au sein de la profession les incite à postuler à la rédaction qui facilite les déplacements, les relations privilégiées comme l'écriture, bref, qui permet d'être un personnage public. Ceci dit, le nombre de places disponibles est inférieur aux demandes adressées, d'autant que les rédacteurs issus des écoles de journalisme abondent, sont mieux formés et moins onércux. Rúsultat : de w.ls avancements se comptent sur les doigts d'une main.

Les journalistes des Pyrénées-Atlantiques pcinent aus:i à parvenir au secrétariat de rédaction, cette fonction exigcant une maitrisc technique spécifique. Son exercice implique de savoir xélectionner, corriger, récrire les articles mais aussi d'élaborer des maquettes papier puis informatisées à partir des textes, photographies et infographies disponibles suivant l'actualité. L'avènement de l'informatique a profondément altéré le secrétariat de rédaction au point qu'un rédacteur ne l'exerçant point régulièrement ou n'ayant pas suivi de stage de formation continue est incapable de s'y retrouver. Les titres hésitent même à confier cette tâche aux jeunes stagiaires car l'enseignement des bases est non seulement long et coûteux mais en plus suppose de désigner un formateur qui ne pourra plus effectuer son propre traváil

Les candidats ont du mal enfin à devenir JRI, d'autant que la concurrence est féroce comme l'agence de télévision ETV en a fait l'expérience. Tandis qu'elle jouissait d'un contrat avec Euskal Telebista, la chaîne publique a décidé de le rompre et de lancir un appet d'offre sur l'ensemble du Pays Basque, de part et d'autre de ta frontière. Les conditions imposées sur la disponibilité, le temps de travail et la rémunération étant des plus restrictives, ETV n’était plus compétitive. Le marché est emportê par une entreprise rivale, dont le siège se trouve outre-Bidassoa, qui fournit les images à Iparraldearen Orena et dont les cameramant: auviènt en coopération avec les rédacteurs d'ETB. Finalement, ETV a licencié ses journalistes reporters d'images et son directeur peine à trouver des piges.

II Entretien avec un rédacteur pigiste. 
La rivalité est d'autant plus rude que le nombre de télévisions dans les PyrénéesAtlantiques est restreint. Le département ne dénombre que deux chaînes locales, France 3 Pays Basque et Euskal Telebisia lparralde en l'occurrence, et deux bureaux détachés de France 3 Aquitaine. Au total, elles comptent huit JRI titulaires et deux ou trois JRI pigistes. Ces derniers, béarnais de surcroît, sont mal lotis car les opportunités de travail s'amenuisent comme peau de chagrin au point de les contraindre à se déplacer au Pays Basque, dans les Landes, dans les Hautes-Pyrénées voire dans le Lot-et-Garonne. La situation n'est guère comparable à celle de la région parisienne et des métropoles régionales où se trouvent les sièges des différentes chaînes généralistes et spécialisées sans compter les agences de télévisions privées.

Ainsi, pour quantité de candidats, le songe est fugace dans la mesure où ils renoncent au jollnalisme reportage d'images eu égard aux complications inhérentes à cette fonction. Leur choix s'est porté sur la photographie parce qu'ils estiment que leurs chances s'en trouveraient accrues. "A mes débuts, j'ai voulu ètre JRI. Étant attiré par l'image, animée de surcroît, je trouvais qu'elle offrait de grandes potentialités car l'image est beaucoup plus puissante et efficace que le texte pour traduire les émotions, les sentiments, bref, le vécu. Mais j’ai rapidement fait une croix dessus et me suis orienté vers la photographie qui m'autorisait à rester dans cet univers" 12 .

Entin, laborantins, titulaires d'un CAP photo ou personnes extérieures à la profession qui arrivent au photo-journalisme sont rares actuellement. Les reporters titulaires ou pigistes de Ia presse quotidienne et hebdomadaire, du Béarn et du Pays Basque, de magazines et d'agences de presse, représentent un nombre suffisant eu égard au marché. Chaque média dispose dé ses reporters attitrés et puise parmi les indépendants lorsque l'actualité se densifie et s'amoncelle. "Toutes les places sont prises, d'autant que le marché départemental est réduit. En somme : les quelques photographes indépendants éprouvent les pires difficultés pour obtenir la moindre pige et certains galèrent en ne parvenant pas à joindre les deux bouts" 13 .

\section{Fragilité des statuts}

S'y ajoute une fragilisation des statuts avec l'accroissement des contrats précaires (CDD, CES, Contrats de Qualification, Contrats Commerciaux), des temps partiels ${ }^{14}$, du pigisme comme du chômage qui affectent particulièrement les femmes, les jeunes et les quinquagénaires, les moins diplômés.

12 Entretien avec un reporter-photographe.

13 Entretien avec un photographe indépendant.

14 Audric. S, Forgeot. G., "Le développement du travail à temps partiel". Paris, Données Sociales, INSEE, 1999. 


\subsection{Développement des contrats précaires}

Les contrats précaires se multiplient depuis les années 80 , à l'instar des CDD. Sur l'ensemble de l'Hexagone, les précaires (CDD et pigistes) augmentent de $38 \%$ en six ans allant de 4100 en 1993 à 5650 en 1999, leur pourcentage passant de $15 \%$ à $19 \%$ des journalistes. Idem dans les Pyrénées-Atlantiques, d'autant que les départements frontaliers et côtiers sont attractifs. De la sorte, les précaires sont passés de 28 en 1996 à 33 en 1999, soit $18 \%$ de la profession. "Encore faut-il préciser que ce total est largement minoré tant les obstacles sont grands sur la route de l'obtention du sésame de la carte. De l'ignorance du statut en passant par les moyennes de piges insuffisantes (plus proches du RMI que du SMIC) sans oublier les invites patronales à différer la demande quand le statut du correspondant local n'est pas proposé" 15.

Le recours aux Contrats Emploi Solidarité (CES) s'est aussi amplifié 16 . Alors qu'ils n'existaient point (le plan pour l'emploi des jeunes date de 1986, te décret pour la réinsertion des chômeurs de longue durée de 1985 et le plan pour l'emploi de 1988) ou n'étaient pas employés par l'audiovisuel public, Sud Ouest, ni Pyrénées Presse. les TUC-CES sont devenus la norme pour nombre de professionnels. Souvent, le provisoire dure et les journalistes entreprennent une carrière précaire qui les mène d'un CES à un Emploi Jeune en transitant par un CEC et un Contrat de Qualification.

Les bénéficiaires des CES sont les jeunes de 18 à 25 ans, les titulaires au plus d'un CAP-BEP, les demandeurs d'emploi inscrits pendant 12 mois au cours des 18 mois précédant la date d'embauche, les personnes percevant l'allocation de fin de droits ou l'allocation de solidarité spécifique, les titulaires du RMI et, à titre exceptionnel, des personnes ne remplissant pas ces conditions mais rencontrant des tracas particuliers d'accès à l'emploi. La durée de ces contrats oscille entre 3 et 12 mois, pouvant atteindre 24 mois, et suppose un investissement hebdomadaire d'au moins 20 heures. Ces contrats sont fort avantageux pour les médias, l'État prenant en charge la totalité ou la majeure partie $(8.5 \%)$ de la rémunération des salariés ainsi que les frais de formation complémentairus sur une base forfaitaire, tout en étant exonérés des charges patronales. En réalité, les CES concernent un public bien plus ample, les contrats aidés étant détournés de leur vocation initiale.

Les radios locales privées y ont spécialement recours pour assurer leur constitution puis leur survie, à l'exemple de Radio Oloron qui utilise régulièrement les CES dans la mesure où, actuellement, la station compte deux personnes en CES auxquelles s'ajoutent trois individus en CEC dont deux à 130 heures par mois ut une à 87 heures par mois. Ces contrats étant

15 Le Journaliste, 2è trimestre 1999.

16 Belloc. B, Lagatrenne. C., "Emplois temporaires et emplois aidés". Paris, Données Sociates, INSEE, 1996 
de courte durée, le renouvellement du personnel est fréquent, malgré les efforts de la direction pour le garder. Depuis 1992, la radio recrute trois à quatre CES par an et les conditions d'accès à ces contrats sont telles que la station emploie avant tour des jeunes non qualifíés, des chômeurs de longue durée voire des RMIstes.

L'usage du contrat de qualification a également augmenté compte tenu du fait qu'il assure une prise en charge partielle de la rémunération, des frais de formation et des charges patronales par l'État (encouragé par des mesures gouvernementales d'insertion et de réinsertion dans le marché de l'emploi telles que le Plan Avenir Jeune de 1981, le Plan pour l'emploi des jeunes de 1986, le plan pour l'emploi de 1988). Les médias, dont les radios locales privées et les quotidiens transfrontaliers, l'utilisent sans retenue et même les principaux titres sont tentés d'employer de jeunes recrues par ce biais, ce qui est rentable pour le titre et qualifiant pour le journaliste.

La rédactrice de Gara qui dispose d'un tel contrat en est l'illustration. "Je suis entrée à Gara comme journaliste stagiaire afin de couvrir l'actualité du Pays Basque nord tout en étant formée par mon collègue. Les mois passant, je me suis retrouvée sans véritable contrat de tritvail ni couverture sociale. Nous avons donc demandé que ma situation soit régularisée aux yeux des législations française et espagnole. Après plusieurs semaines de discussion, nous sommes parvenus à un accord stipulant que je disposais d'un contrat de qualification durant deux ans qui me permet de conjuguer travail salarié et formation professionnelle. L'atribution du contrat a été tardive, la direction cherchant à profiter des opportunités olfertes par la loi" 17.

D'autres journalistes sont davantage concernés par les contrats commerciaux qui sont usés par les médias transfrontaliers, à savoir Egin puis Gara, Radio Euskadi, Euskadi Irratia et Euskal Telebisia. Sans équivalent en France, ils sont décernés aux salariés pour une période n'excédant pas douze mois puis reconduits à l'envi. Comme le dit cette rédactrice, "mon contrat dè travail est renouvelé tacitement chaque année par le quotidien Egin. Mais il suffit que les ventes déclinent, que les recettes publicitaires chutent ou qu'un juge ferme temporairement ou définitivement le journal pour me retrouver sans emploi. Egin a ainsi procédé à des liceñcièments au début des années 90 dans un contexte de crise publicitaire" 18.

Comment comprendre cet accroissement des contrats précaires? Premièrement, outre la volonté croissante des médias de réduire les dépenses de personnel, il s'explique par le fait que ces journalistes ont un niveau de qualification inférieur à celui de leurs homologues nationaux. Ainsi, deux des trois photographes pigistes de Pyrénées Presse disposent d'un CAP quand le troisième est bachelier. Les rédacteurs de Xiberoko Botza et des radios municipales béarnaises ne sont pas diplômés d'écoles de journalisme. Dès lors, ils sont condamnés à ent-

17 Propos d'une rédactrice de Gara.

18 Entretien avec la rédactrice d’Egin. 
rer dans la profession par la petite porte, synonyme de CES, de contrats de qualification ou de contrats commerciaux alors que ceux issus de ces écoles obtiennent immédiatement des stages, des CDD puis des CDI.

Deuxièmement, cela fait suite au manque d'expérience des journalistes novices dans la profession. Les médias préferent leur concéder des contrats précaires parce qu'ils ne disposent guère des savoirs, savoir-faire, savoir-dire et savoir-être exigés par l'exercice du journalisme local. D'un côté, le jeune a l'occasion d'acquérir une expérience et de faire la preuve de ses compétences professionnelles. D'un autre côté, l'employeur dispose d'une main d'œuvre bon marché qui, si elle est performante, se verra proposer un contrat en bonne et due forme.

Troisièmement, cela résulte de la volonté des entreprises de presse d'opérer une meilleure utilisation du temps. "Lorsque l'activité connaît des fluctuations saisonnières ou lorsqu'il faut honorer des commandes imprévues, il est facile de faire appel à ce type de main d'œuvre. Ainsi, l'entreprise économise sur les temps morts en ajustant au plus près le travail rémun $r$ au travaii effectif. Les entreprises font appel en général à l'intérim pour un besoin brel, au CDD pour un besoin prévu, régulier et plus long" 19

\subsection{Prolifération des temps partiels}

Les temps partiels foisonnent aussi à partir des années 80 , particulièrement auprès des femmes et des jeunes journalistes qui représentent $70 \%$ des temps partiels. Les radios locales privées y font amplement appel, à l'image de Gure Irratia ou la totalité da animbatcurs et trois journalistes sur quatre pâtissent de temps partiels $(20 \mathrm{~h}, 25 \mathrm{~h}, 35 \mathrm{~h})$ et d'Irulegiko Irratia qui employait ses quatre reporters-présentateurs à temps. Le service public n'est guère épargné pui que deux des huit jutimalistes de France 3 Pays Basque ceuvrent à mi-temps, France Blett Pays Basque offrant un panorama comparable.

D'abord, le trois-quart temps se généralise dans les Pyrénées-Atlantiques car l'ensemble des supports l'utilise la presse, la radio comme la télévision ainsi qu'un grand nombre de médias, de Curre Irratia à La Semaine du Pays Basque en passant par La République des Pyrénées. Ainsi, le photographe de l'hebdomadaire et deux des quatre reporters de la station locale associative sont à trois-quart temps, permettant de concilier les emplois d'un rédacteur et d'un secrétaire de rédaction, d'un journaliste et d'un animateur, d'un rédacteur et d'un JRI. Si le trois-quart temps accorde vie privée et vie professionnelle, surtout pour les femmes mariées avec enfants à charge, il est préjudiciable professionnellement car synonyme de salaire limité et de stagnation de carrière ${ }^{20}$.

19 Paugam. S. Le salarié de la précarité. p.71, Paris, PUF, 2000.

20 Nicole-Drancourt. C., Carrières masculines, carrières fêminines. Paris, CNAF, 1988. 
Ensuite, le mi-temps connaît un développement notoire aussi bien au Pays Basque qu'en Béarn dans la mesure où il concerne surtout les médias locaux, dont les locales de Radio France et France 3 Pays Basque. Les femmes et les jeunes figurent en bonne place parmi les bénéficiaires du mi-temps alors que les hommes de plus de quarante ans sont peu concernés. S'il est parfois choisi afin de consacrer davantage de temps à leur famille, à leurs loisirs voire à leur santé, il est souvent subi. Qu'il émane de la direction ou d'une décision collégiale, tel que cela s'opère dans les radios d'expression basque, il est rarement conforme au souhait de la pirsunne concernée.

Enfin, le temps de travail indéterminé se propage avec l'avènement du pigisme de masse. Il est restreint sans pouvoir préciser sa durée tant les situations sont diverses et fluctuantes. D'un journaliste à l'autre, d'un mois à l'autre, d'un entreprise de presse à l'autre, le temps de travail change. De dix à trente-cinq heures par semaine, de deux à quatre jours de travail hebdomadaire, de trois à dix heures par jour. Ne figurant sur aucun document, il varie suivant les CDD, les piges journalières et les articles rédigés. Or, il parvient difficilement à quarante heures tant les piges sont insuffisantes avec l'augmentation des journalistes, d'une part, et la diminution des piges pour indépendants, d'autre part. Deux cas de figure se présentent.

Premièrement, les journalistes ceuvrant à la journée, tel ce photographe pigiste de Prrénées Presse. "En moyenne, mon temps de travail hebdomadaire équivaut à deux jours complets. Il peut aussi bien s'agir du lundi et du mercredi, du mardi et du vendredi ou du week-end. Cela est tributaire de la densité de l'actualité, de la disponibilité des autres indépendants (étant le plus jeune et le dernier arrivé), du budget piges disponible. Ponctuellement, le chef du service photo de Pyrénées Presse me propose d'oeuvrer jusqu'à cinq jours par semaine. Cette semaine, par exemple, j'ai travaillé lundi, mardi, mercredi, samedi et dimanche. Dans tous les cas, les journées débutent à 9 h30 et se terminent à 21 h et, lorsque je suis de permanence, elles peuvent se prolonger jusqu'à $2 \mathrm{~h}$ du matin" 21.

Deuxièmement, les journalistes rémunérés à l'article n'ont pas de temps de travail précis, telle cette correspondante du Diario Vasco. "Je n'ai pas de temps de travail quotidien ni hebdomadaire. Mes horaires fluctuent fortement d'une semaine à l'autre suivant l'actualité, le nombre et la longueur des articles à rédiger, les demandes des chefs de service. En moyenne, cela représente vingt-cinq heures par semaine, soit deux à six heures par jour. Dans ce laps de temps, je lis la presse, je passe des appels téléphoniques, je me documente, je me rends à des conférences de presse ou à des manifestations, je rédige les articles. Mon temps de labeur actuel est inférieur à ce qu'il était au début dans la mesure où, l'expérience aidant, j'ai constitué des dossiers, j'ai une meilleure mâtrise des sujets, je travaille davantage par téléphone et je rédige plus rapidement" 22.

21 Entretien avec un reporter pigiste à Pyrénées Presse.

22 Entretien avec la correspondante du Diario Vasco. 
Le recours massif au temps partiel a divers fondements. D'une part, il accroît la productivité des journalistes compte tenu du fait que, œuvrant vingt à trente heures par semaine, ils ne sont point affectés par la fatigue et le surmenage. Ils se consacrent entièrement à leur tâche et perdent moins de temps en pause-café et en débats représentant plusieurs heures par semaine. Plus encore, ayant une vie privée plus dense sur les plans familial comme des loisirs (lectures d'ouvrages, visionnages de films, activités sportives), ils sont plus à même de proposer des sujets originaux et novateurs. Les discussions alimentées ou écoutées à l'improviste dans un café ou un marché concourent à ce renouvellement. Leurs angles témoignent des moments passés à observer voire à scruter des acteurs de la vie politique, économique, culturelle et les scènes de la vie quotidienne dans une banque, une fête de village ou un supermarché.

D'autre part, cette forme de sous-emploi accrô̂t les fieures travaillées puisqu'un journaliste disposant d'un trois-quart temps œuvre, en fait, l'équivalent d'un temps plein. Comme le relate cette rédactrice, "je bénéficie d'un trois-quart temps à Irulegiko Irratia alors que j'opère environ quarante heures par semaine. Au terme de mon temps de travail légal, je reste au studio afin de monter un reportage, avancer la bi:snı̣ne du lendemain, aider un collègue à réaliser son sujet ou ranger les dossiers; d'autant que les eflectifs sont réduits. Mais, ce travail additionnel n'est pas rémunéré sous la forme d'heures supplémentaires" 23. Cette pratique s'est vite généralisée car la culture professionnelle valorise l'investissement, la pressence, l'absence d'horaires. Seule l'actualité compte ct toute tentative de respecter les horaires se heurte à la réprobation de la direction voire des colligues.

\subsection{Accroissement du pigisme}

"On assiste à un accroissement considérable du pourcentage de pigistes áu sein de la profession, dont témoignent les statistiques de la Commission de la carte. Longtemps compris en desșous des $8 \%$ de l'ensemble des professionnels, au temps du plein emploi, il s'emballe brusquement dans les années $80: 9,6 \%$ en $1980,12,1 \%$ en $1985,14,7 \%$ en 1990 , (26,8\% en 1995) 24 . Signe de précarité, la pige est d'abord pratiquée par les jeunes et les femmes : $31,7 \%$ des pigistes étaient des femmes en 1981, 40,5\% en 1990" 25. Les reporters-photographes sont particulièrement touchés passant de $16,7 \%$ en 1955 à $24,4 \%$ en 1970 pour atteindre $44,1 \%$ en 1990. La situation ne semble guère s'améliorer puisque l'on dénombre 5501 pigistes au niveau national, 123 au niveau régional et plus d'une trentaine au niveau départemental. Pis

\footnotetext{
23 Intretien aves une idactrice d'Irulegiko Irratic.

24 Balbastre. G., "Une information précaire". p.76, Actes de la recherche en scicuces sociales. $\mathrm{n}^{\circ} 131-132,2000$.

25 Delporte. C., Histoire du journalisme et des journalistes en France. p.87. Paris. PLF 1995.
} 
encore, sur 1880 nouvelles cartes de journalistes délivrées en 1998, 846 (environ 40\%) sont pigistes ou chômeurs. 26

En réalité, les chiffres sont biẹn supérieurs. "On peut supposer que la vraie valeur est plus importante. Ainsi, nombre de pigistes travaillent pour la presse institutionnelle ou d'entreprise en même temps qu'ils pigent pour la presse magazine grand public ou spécialisée. Or. leurs revenus étant constitués pour plus de $50 \%$ par le secteur communication, ils ne peuvent plus prétendre à la carte de journaliste. Il en va de même dans la presse audiovisuelle, où un certain nombre de pigistes relèvent du régime des intermittents du spectacle pour bénéficier de meilleures allocations aux Assedics et n'ont plus de carte. Enfin, d'autres pigistes touchent des sommes insuffisantes pour prétendre à cette fameuse carte (par exemple un certain nombre de correspondants de la presse quotidienne ou hebdomadaire régionale)".

Plus en détail, la presse écrite du département observe un essor notable du pigisme. la totalité des titres étant affectée. Dans le seul service photographique de Pyrénées Presse comprenant trois titulaires, ['on énumère autant de photographes pigistes réguliers auxquels s'ajoutent des indépendants occasionnels. Les quotidiens transfrontaliers font appel à un photographe pigiste pour opérer l'ensemble des clichés et à des rédacteurs indépendants afin de remplacer les titulaires absents. Sans compter les centaines de correspondants, 330 pour Plrénées Presse et 300 pour Sud Ouest Béarn et Soule, chargés de couvrir l'actualité de leur village et leur canton qui opèrent un travail comparable à celui des pigistes sans jouir ni de leur statut ni de leur rémunération.

L'intérim connait aussi une extension nouvelle dans la radiodiffusion des PyrénéesAtlantiques, notamment dans les locales de Radio France qui ne font guère exception. Pis encore, elles se trouvent à l'avant-garde du mouvement dénombrant davantage de pigistes que les radios locales privées pourtant décriées pour la fragilité des statuts octroyés. Ce journaliste illustre le phénomène. "Je suis indépendant depuis de nombreuses années à Pau où j'ai obtenu maintes piges dans le bureau palois de France 3 Aquitaine et la locale de France Bleu Béarn. Étant inscrit dans leur planning local, je suis appelé dès qu'un rédacteur s'absente, l'actualité se densifie et un événement sportif d'importance survient. Étant spécialisé en sport, je suis contacté pour couvrir une rencontre de l'Élan Béarnais, de la Section Paloise et le Grand Prix de. Formule 3 de Pau. Les piges vont d'un jour à plusieurs semaines et leur dotation dépend des saisons, des ressources et des effectifs" 27.

Le pigisme de télévision s'accroît tout autant, notamment à France 3 Pays Basque où l'on dénombre cinq indépendants réguiiers ainsi que des collaborateurs sporadiques en provenance de stations de France 3, alors que la rédaction de France 3 Pays Basque compte sept

26 Jourdain. C., "L'intérim, une voie d'accès à l'emploi". Paris, Données Sociales, INSEE, 1999.

27 Entretien avec un pigiste de l'audiovisuel. 
titulaires. La situation n'est pas meilleure à ETB Iparralde puisque les deux rédacteurs titulaires et les cameramans disposent de contrats commerciaux de neuf mois.

Rendre compte de cette évolution suppose de considérer, d'abord, que le pigisme autorise la baisse des frais de personnel. "Pour échapper aux garanties salariales conventionnelles, ces entreprises (...) recourent de plus en plus à des collaborations extérieures, essentiellement des pigistes dont le nombre est en constante augmentation. La pige (...) est devenue un véritable système de sous-traitance qui permet aux entreprises de presse de réaliser des économies substantielles. Les pigistes ne disposent pas, en effet, de bureaux dans les locaux des journaux, sont payés à la tâche et peuvent être mobilisés ponctuellement. Par contre, ils duivent supporter des coûts d'équipement individuel de plus en plus lourds : fax. télíphone portable pour pouvoir être joints à tout moment par les rédactions, micro-ordinateur, modem et imprimante. De plus, ces petits producteurs individuels doivent tout faire eux-mêmes, ne sont payés qu'après parution de leurs articles, les délais, fait significatif, pouvant aller de 7 à 90 jours" 28.

Ensuite, l'intérim "présente également l'avantage de mettre à la disposition des entreprises de presse des journalistes qui se plient aux lignes rédactionnelles. En effet, parce que la plupart des pigistes se trouvent dans une situation précaire. (...) ils ne peuvent être que dociles vis-à-vis des commanditaires et fabriquent, sans état d'âme, les articles souhaités par les journaux qui les emploient. La rédaction d'articles de compläisance ou de publicité rédactionnelle non avouée comme telle (par exemple la promotion d'un film en échange de places gratuites à distribuer par le journal à ses lecteurs), qui pourrait poser problème au sein des rédactions si elle était discutée en conférence de rédaction et confiée à des journalistes salariés, peut être sous-traitée par des pigistes qui ne sont pas en mesure de soulever des problèmes déontologiques" 29.

Entin, le pigisme offre plus de souplesse et d'élasticité aux entreprises de presse. Fonctionnant peu à peu en flux tendu ${ }^{30}$, les médias du département sont tributaires des repos hebdomadaires, congés, stages dans le cadre de la formation continue. Plus encore, les maladies subites ou les problèmes familiaux génèrent un besoin de main d'œuvre qui n'est guère disponible immédiatement. A Sud Ouest Béarn et Soule, par exemple, les rédacteurs absents ne sont point remplacés, générant un surcrôt de travail. Pareillement, la forte densité de l'actualité locale allant de la visite d'un ministre à un fait divers sanglant en passant par l'implantation d'une entreprise génératrice de dizaines d'emplois suppose l'aide d'un ou de deux reporters supplémentaires. Dès lors, le recours à l'interim devient la solution dans la mesure où les pigis-

28 Champagne. P., "Le journalisme à l'économie". Actes de la Recherche en Sciences Sociales. $n^{\circ} 131-132,2000$, p.5.

29 Champagne. P., idem. p.5-6. Actes de la Recherche en Sciences Sociales, n'131-132, 2000.

30 Amar. M., "Les effets du tlux tendu". L'Entreprise, n²5I8, 1992. 
tes locaux sont promptement disponibles, rompus aux techniques spécifiques de chaque fonction et connaisseurs des acteurs comme des problématiques locales.

\subsection{Essor du chômage}

Sans surprise, le chômage de ces journalistes n'a cessé de grandir au cours des années 80 et 90 passant de $0,4 \%$ en 1970 à $3,1 \%$ en 1990. Là encore, il vá bien au-delà dans les faits. D'abord, "la carte de presse est refusée au-delà de deux années de chômage, si aucune pige n'a été faite ni aucune formation entreprise" 31 . Ensuite, de nombreux sans-emplois ne signalent leur siluation ni à la Commission de la Carte ni à l'ANPE autant par honte que par espoir de trouver quelques "papiers" les aidant à vivre de leur activité professionnelle. Enfin, la réalisation de quelques piges octroie un revenu inférieur à 3000 francs écarte les journalistes de la catégorie chômeur. Ce que confirme Delporte : "la pige permet de limiter les effets du chômage. Les entreprises de presse disposent alors d'une main d'œuvre souple à gérer" 32.

Certains publics sont singulièrement touchés, à l'tmage des jeunes journalistes ${ }^{33}$. Qu'ils œuvrent dans la presse, la radio ou la télévision, le constat est similaire. Souvent dépourvus de diplômes reconnus, ils peinent à trouver des CDD voire des piges. Méconnus des confrères comme des employeurs, ils ne disposent guère des réseaux relationnels susceptibles d'être mobilisés afin de mettre le pied à l'étrier. Cela pousse les uns à réaliser des stages gratuitement afin de fuire montre de leurs compétences mais. à leur terme, le rédacteur en chef les remercie sans contrat. Les autres envisagent de quitter le département à la recherche de régions plus propices. Lorsqu'ils obtiennent un voire plusieurs contrats à durée déterminée et que l'entreprise connaît des difficultés, ils en sont les premières victimes.

Ce rédacteur de presse locale hebdomadaire en offre l'exemple. "Mon parcours professionnel a connu des interruptions synonymes de chômage car, entre ma sortie de l'école de journalisme en juin 1998 et mon arrivée à La Semaine du Pays Basque en février 1996. vingt mois se sont écoulés. Période au cours de laquelle j'ai alterné dix mois de CDD avec autant de temps d'inactivité. Le chômage est difficile à vivre car si cela est supportable durant les premiers mois, fa stulation se dégrade à partir du sixiène mois. On se sent mal à l'aise dans les conversations avec autrui même si je n'ai jamais douté de moi sachant que des opportunités se présenteraient. C'est pourquoi j'ai continué à envoyer des curriculum vitae. Cela a été faciitité par le soutien de ma famille" 34 .

31 Profession journaliste $\mathrm{n}^{\circ} 289$, mai-juin 1999.

32 Delporte. C., idem. Paris, PUF, 1995.

33 Baudelot. C, Establet. R., Avoir 30 ans en 1968 et en 1998. Paris, Seuil, 2000.

34 Entretien avec un rédacteur de la Semaine du Pays Basque. 
Ensuite, les quinquagénaires sont aussi visés, d'autant qu'ils sont confrontés au chômage sans y être préparés s'agissant d'entants des "trente glorieuses" 35. Les journalistes cuvrant dans les radios locales municipales et associatives ainsi que dans les quotidiens tranfrontaliers sont fort concernés puisque les trois rédacteurs en chef de Radio Bayonne, Radio Pais et Egin en ont fait l'expérience. Leur licenciement résulte de la disparition des médias. dans lesquels ils occupaient des postes à responsabilité, ou du souci de réduire les frais de persomel. Retrouver un emploi suppose de renoncer à l'encadrement et d'accepter de repartir comme rédacteur de base.

En outre, les femmes sont très affectées par le chômage indépendamment des médias, des postes et des fonctions. Sur les $40 \%$ des pigistes de sexe féminin, nombreuses sont celles oscillant entre la pige cî le chômagc. Les employeurs rechignent à les recrutcr par crainte que leur condition féminine ne porte préjudice à l'entreprise car elless seraient plus souvent absentes, moins présentes le soir, trop ́́notives. Les femmes seraient moins agressives dans la recherche d'un emploi et guère prêtes à tout pour y parvenir, leur vie privée occupant une place de choix. Les directeurs perçoivent souvent les sphères familiales et professionnelles comme antagoniques alors qu'elles sont jugées complémentaires pour les hommes.

La perte d'emploi entraine des tracas financiers, surtout lorsque les membres de la gente féminine sont divorcées avec enfants à charge. "Les fins de mois étaient souvent rudes et nous devions faire des sacrifices dans les loisirs, les vêtements, la nourriture. Les enfants ne comprenaient pas toujours que nous ne puissions pas partir en vacances ou acheter un jouet dont ils avaient follement envie. Nous allions voir des assistantes sociates afin d'obtenir des aides à destination des personnes en difticulté, dont le RMI, et des aides spécifiques aux familles monoparentales" 36. Les célibataires, sans enfant et vivamt maritalement, s'en sortent mieux parce que le salaire de leur conjoint associé à leurs indemnités suffit à assurer un niveau de vie convenable.

Enfin, les journalistes peu diplômés sont tout aussi concernés par ce drame étant deux à trois fois plus nombreux à en pâtir que ceux ayant transité par une école de journalisme reconnue par la convention collective. Être dépourvu de diplômes de l'enseignement supérieur est synonyme de culture (technique et non génćrale), de manière de penser (anecdotique et non systématique), de style d'écriture (littéraire et non journalistique) inadaptés. Plus encore, ils sont privés des connaissances nouées durant les études, des pratiques culturelles et des loisirs susceptibles d'être mobilisés pour la recherche d'un emploi.

Ce rédacteur bachelier l'illustri puisqu'il a été confronté au chômage à plusieurs reprises. Une première fois, lorsque le CDD de trois mois dont il disposait au Berry Républicaint n'a pas été renouvelé suite à un changement de direction puis à sa volonté de placer ses hom-

35 Fourastié. J., Les trente glorieutes. Paris, Wuyard, 1979.

36 Entretien avec une pigiste. 
mes de confiance. Une deuxième fois, quand Pyrénées Presse a décidé de le licencier après avoir multiplié les contrats à durée indéterminée durant un an. Le titre devait choisir entre l'intégrer ou le congédier, car l'inspection du travail risquait d'y mettre son nez. Une troisième fois lorsqu'il est venu au Pays Basque dans l'espoir de trouver un emploi fixe ou, à défaut, de piger pour les médias locaux et régionaux. Il a littéralement. "galéré" durant un an, entre son CDD de quatre mois au Midi Olympique en 1994 et sa correspondance pour Sud Ouest en 1995.

L'augmentation du chômage est liée, en premier lieu, à la fonction primordiale des Écoles de journalisme excluant ceux qui n'y entrent point. En effet, le rôle des établissements reconnus par la convention collective de la profession est essentiel dans la recherche d'emploi. Les personnes qui sortent de l'ESJ, du CFJ et, dans une moindre mesure, des IUT, ont beaucoup plus de chances de trouver un emploi parce que ces institutions ont noué des conventions avec les principaux médias nationaux, régionaux voire locaux. Cela les aide à entreprendre des stages prolongés dans les titres de leur choix et à constituer des carnets d'adresses. Alors que celles n'y transitant pas éprouvent les pires résistances pour trouver le moindre stage et sont confrontées au chômage.

En second lieu, le chômage résulte de l'augmentation substantielle des journalistes professionnels car chaque année, des centaines d'étudiants sortent des SIC de Bordeaux, de Leioa et des écoles spécialisées. Ils sont de plus en plus nombreux à prétendre à des postes limités en quantité dans les médias locaux et régionaux. L'offre étant supérieure à la demande, certains d'entre eux connaissent le chômage qui est bien plus important que ne le laissent croire les chiffres divulgués par la profession car certains professionnels sont contraints de quitter momentanément voire définitivement leur métier pour travailler dans les services de commerce et de communication.

En dernier lieu, la raison réside dans la fragilité financière des entreprises de presse consécutive à leur dépendance face aux débiteurs, à savoir des annonceurs, le Fond de Soutien à I'Expression Radiophonique, le Conseil Général, les mairies ou les associations. Il suffit qu'une aide soit abrogée ou qu'une subvention tarde pour placer la radio dans une situation délicate. Lorsque la subvention supprimée est d'une grande ampleur, les radios municipales, surtout, éprouvent les pires embarras pour trouver des recettes annexes, d'autant qu'elles ne peuvent souvent compter sur la mobilisation des auditeurs pour infléchir la décision des institutions ou combler le déficit. Cela donne lieu à la disparition pure et simple de la station ou à sa reprise par un réseau national intéressé par sa fréquence. Dans les deux cas, cela débouche sur des licenciements. 


\section{Conclusion}

Rappelons que cet article défend l'hypothèse selon laquelle, depuis les années 70, la condition journalistique locale connaît une précarité grandissante avec la faible progression de carrière, la fragilité des statuts et la modestie des revenus, bien que nous nous soyons contenté d'évoquer les deux premières dimensions. Plus en détail, à la promotion réduite de postes, d'échelons et de fonctions se joint la fragilité des statuts avec l'expansion des contrats précaires, du pigisme, des temps partiels comme du chômage. Le sous-emploi est le plus souvent subi par les journalistes, même si certaines femmes le choisissent afin de concilier vie professionnelle et vie familiale, alors que l'absence d'emploi peut conduire à la misère, à la marginalité voire à la désaffiliation ${ }^{37}$. La précarité affecte le centre même de la profession, même si ses effets sont plus perceptibles à ses marges.

Au-delà, cette précarité s'inscrit dans la fragilisation de la profession observable dans les annêes 80 et 90 consécutivement à la multiplication et à la diversification des systèmes médiatiques et journalistiques locaux, et se manifeste tout autant dans les dérives de la pratique. D'abord, l'accès aux sources d'information politique, économique et médiatique est parfois malaisé car si les grands titres font aussi bien appel aux agences de presse, aux élus, aux entreprises qu'aux institutions locales, tel n'est point forcément le cas des nouveaux médias. Ensuite, les journalistes disposent d'une autonomie sous contraintes qui circonscrivent leur liberté dans le choix des sujets, des angles, de la hiérarchisation et de l'emplacement de l'information devant faire face aux impératifs économiques et techniques entre autres. Enfin, la rigueur fait quelquefois défaut, l'information n'étant pas systématiquement vérifiée et l'impartialité comme la neutralité étant parfois bafouées.

Nonobstant, cet affaiblissement n'est point synonyme de crise sérieuse de la profession journalistique Iocale. Loin d'être la preuve d'une dégradation profonde et durable au regard d'un passé glorifié et d'un présent dramatisé, il indique un ralentissement voire un arrêt momentané du processus de professionnalisation à l'œuvre depuis l'entre-deux-guerres. Cette professionnalisation s'est traduite par la dotation d'un statut professionnel, d'une convention collective, d'une organisation syndicale, d'un code de déontologie, d'une protection légale du monopole, bref, de tout ce qui définit une profession au sens anglo-saxon ${ }^{38}$ du terme.

D'autant que la fragilisation est partiellement compensée par l'apparition ou le renforcement de régulateurs. Ainsi, au cours des années 90 , les relations professionnelles se redynamisent avec l'explosion des organisations comme des mobilisations professionnelles. D'un côté, aux syndicats de journalistes se joignent des associations spécialisées qui jouissent d'une

37 Castel. R., Les métamorphoses de la question sociale. Une chronique du salariat. Paris, Fayard, 1995. 38 Wilensky. H., "The Professionalization of Everyone?". American Journal of Sociology, 2, p. 137-158, 1964. 
ample représentativité et qui proposent des actions aussi consensuelles et variées que l'information, le service, le soutien des confrères en difficulté, la pression sur les pouvoirs publics ou l'organisation de débats. D'un autre côté, leurs mobilisations connaissent des processus analogues à l'instar des thèmes (salaires, abattement fiscal de $30 \%, 35$ heures, emploi, programmes locaux, droits d'auteur), des modes d'action (procès, rassemblement, grève, pétition, lobbying, conférence de presse) et des acteurs engagés. 


\section{Bibliographie}

ACCARDO. A., Journalistes précaires. Bordeaux, Le Mascaret, 1998.

AMAR. M., "Les effets du flux tendu". L'Entreprise, n² 2518, 1992.

AUDRIC. S, Forgeot. G., "Le développement du travail à temps partiel". Paris, Données Sociales, INSEE, 1999.

BALBASTRE. G., "Une information précaire". Actes de la Recherche en Sciences Sociales, $n^{\circ} 131-132,2000$.

Baudelot. C, Establet. R., Avoir 30 ans en 1968 et en 1998. Paris, Seuil, 2000.

BELLOC. B, Lagarenne. C., "Emplois temporaires et emplois aidés". Paris, Données Sociales, INSEE, 1996.

BOLTANSKI. L, Chiapello. E., Le nouvel esprit du capitalisme. Paris, Gallimard, 1999.

CASTEL. R. Les métamorphoses de la question sociale. Une chronique du salariat. Paris. Fayard. 1995

CaVAlles. T. Radio France Pau Béarn. Paris, Sport. Com, 1999.

CHAMPAGNE. P., "Le journatisme à l'úcunomie". Actes de la Recherche en Scienci" Sociciles, $n^{\circ} 131-132,2000$

CHARON. J-M., Carte de presse. Paris, Stock. 1993.

DAUCÉ. N., "Quand ia précarité redessine le marché de l'emploi". in. Le monde du trunail. Paris, La Découverte, 1998.

DELPORTE. C. Histoire du journalisme et des joumalistes en France. Paris, PUF, 1995.

FOURASTIÉ. J., Les trente glorieuses. Paris, Fayard. 1979

FROUIN. J-Y.. "Les éléments de précurité danns le contrat à durée indéterminée", DO, 125. 1997.

JOSA. V.. La Sancine du Pam Basque. Bordeaux. ISIC. 1995.

JOURDAIN. C., "L'intérim, une voie d'accès à l'emploi". Données Sociales, INSEE, 1999.Le Journaliste, 2ème trimestre 1999.

MATHIEN. M., Les joumalistes. Paris, PUF, 1995.

NICOLE-DRANCOURT. C., Carrières masculines, carrières féminines. Parris, CNAF, 1988.

PAUGAM. S., L'exclusion, l'état des savoirs. Paris, La Découverte, 1996.

PAUGAM. S.. Le salarié de la précarité. Paris, PUF, 2000. 
PROFESSION JOURNALISTE, n²88, décembre 1998-janvier 1999.

PROFESSION JOURNALISTE, n²89, mai-juin 1999.

RIEFFEL. R., Les journalistes français en 1990. Radiographie d'une profession. Paris, La Documentation française, 1991.

URTEAGA. E., Les joumalistes locaux : mutation d'une profession. Thèse de Doctorat en Sociologie réalisée sous la direction de François Dubet, CADIS-CNRS, Université de Bordeaux II, 2000.

URTEAGA. E., "Kazetariak eta heziketa profesional iraunkorra”. Bilbo. Giza eta Gizarte Zientzien Aldizkaria: Uztaro, n³6, pp.53-71, 2001.

WIEVIORKA. M.. La violence en France. Paris, Seuil, 1999.

WILENSKY. H.. "The Professionalization of Everyone?". American Journal of Sociology, ก², pp. 137-158, 1964

WOLTON. D., Penser la communication. Paris. Flammarion. 1997.

ZEGROUIN. A., Radio Bavonne. Bordeaux. ISIC, 1992. 